\title{
The trends in myocardial revascularization
}

\author{
John P. Pirris \\ Department of Surgery, Division of Cardiothoracic Surgery, University of Florida College of Medicine, Jacksonville, USA
}

Attempts to revascularize the heart were made as early as 1935 by direct perfusion. The procedures included transmyocardial acupuncture and implanting internal mammary artery into the myocardium to achieve neovasularization and collateral formation. We now all much more familiar with coronary artery bypass grafting (CABG) and percutaneous transluminal coronary angioplasty (PTCA) or percutaneous coronary intervention (PCI) with stents. The Drug Eluting Stents (DES) reduce the rate of restenosis while they increase the rate of stent related thrombotic events. Surgical techniques of CABG have been constantly changing and improving for over half of a century. Percutaneous transmyocardial revascularization (TMR), off pump CABG, CABG plus adjuvant TMR, and CABG with TMR plus adjuvant gene therapy are the techniques and procedures we are going to be discussed here.

\section{Catheter-based revascularization \\ percutaneous}

\section{myocardial}

Myocardial revascularization with PCI has gone through eras of balloon angioplasty, catheter based laser radiation, bare metal stents, first generation drug eluting stents, second generation drug eluting stents and the newly developed administration of stem cell therapy.

Percutaneous myocardial revascularization was approve to be a safe and feasible therapeutic option in patients with coronary artery disease $(\mathrm{CAD})$ and severe angina pectoris not amenable to either CABG or PTCA towards the end of the last century. Comparison studies between CABG and PCI with balloon angioplasty, bare metal stents or first generation drug eluting stents have shown the rates of major adverse cardiac events (MACE) among multivessel CAD after CABG are lower than PCI stents. The stents reduce the rate of restenosis while they increase the rate of stent related thrombotic events. CABG groups have shown lower long-term mortality with higher risk of stroke. However, the risk of death associated with Everolimus-eluting (second generation) stents was similar to CABG but a lower risk of stroke. PCI with the second generation eluting is still associated with a higher risk of myocardial infarction (MI) with incomplete revascularization and repeat revascularization. Restenosis and stent thrombosis are associated with potentially and significantly increasing in the rates of MI and death.

Systemic review and meta-analysis suggested that the advantage of CABG over PCI was consistent among all trials for the primary end point of MACE and was most pronounced in the high-risk SYNTAX Score group with improved outcomes. In overall population, trials have shown similar outcomes in patients with low to intermediate risk SYNTAX Score with multivessel diseases. It's not the case in the diabetic subgroup patients, however. Superior outcomes with CABG were demonstrated largely related to higher rate of in-stent restenosis and subsequent target vessel revascularization with PCI. The second generation stents reduce event rate in diabetic patients mainly driven by a lower incidence of stent thrombosis and mortality with no significant differences in restenosis. Incomplete revascularization has been associated with a significant higher risk of death, MI and repeat revascularization, and is considered as an independent predictor of MACE and repeat revascularization. The magnitude of risk is much higher in the incompletely revascularized PCI group compared with $\mathrm{CABG}$ group. The major reason for long term durability and improved outcomes of CABG may be related in part to the completeness of revascularization. Therefore, CABG is preferred for the most favorable long term outcomes in high-risk SYNTAX Score patients without high surgical risk.

\section{Off-Pump CABG versus on-Pump CABG}

Off-pump CABG has been around since early 1990s. Comparing with the conventional on-pump CABG, the off-pump approach was meant to potentially avoid cardiopulmonary bypass (CPB) related complications and adverse effects, and to avoid aortic cannulation to decrease the incidence of stoke and other embolic phenomena. Comparison studies have shown reduced short term mortality and morbidity with the greatest relative benefit shown in patients with the most preoperative risk factors. The off-pump CABG is preferred option in older patients, in high risk patients and those with severe atherosclerotic aortic disease.

The use of off-pump procedures reached the peak in 2008(close to $25 \%$ ) with a progressive decline in off-pump frequency since then. A meta-analysis of 22 studies enrolling a total of more than 100,000 patient has demonstrated that the off-pump CABG is likely to have worse long-term (5years or more) survival than on-pump CABG. The on-pump CABG leads to more complete revascularization and increased formation of distal anastomosis. It is a better option in emergency situations and accepted by most surgeons. Enthusiasm for off-pump CABG technique has been tempered.

\section{CABG with adjuvant TMR}

Incomplete revascularization is noted to occur in up to $25 \%$ of CABG surgery due to the severe CAD. TMR emerged as treatment for patients with diffused CAD not amendable to PCI or CABG. TMR improve myocardial perfusion by the creation of laser channels within the ischemic myocardium not amenable to bypass grafting. This hybrid approach may be performed with either on-pump CABG or off-pump CABG. It can be used for regions of myocardial and poor targets or poor conduit.

Correspondence to: John P. Pirris, MD, FACS, Chief of Cardiothoracic Surgery, Department of Surgery, Division of Cardiothoracic Surgery, University of Florida College of Medicine, Jacksonville, USA, Tel: 904383-1011; Fax: 9042443553; E-mail: John.Pirris@jax.ufl.edu

Received: May 30, 2015; Accepted: July 10, 2015; Published: July 13, 2015 
There are two laser devices for TMR are approved by the US Food and Drug Administration: the Novadaq $\mathrm{CO}_{2}$ laser and the Cardio Genesis TMR Holmium laser. There were no differences noted in long-term outcomes for morbidity and mortality between $\mathrm{CO}_{2}$ and Holmium lasers in combination with CABG. The use of TMR in conjunction with CABG surgery is increasing with reduced mortality and MACE. TMR has been show to improve myocardial perfusion by neo-revascularization.

\section{TMR with adjuvant stem cell therapy}

Animal and clinical studies of the combination of stem cell therapy demonstrated that injection of stem cells with TMR was safe with improvement in angina class, in perfusion and left ventricular contractility. Among the different cell studied, autologous bone marrow stem cells were harvested on the same day of surgery and have shown promise in promoting the paracrine secretion of growth factors and cytokines. We are still searching for the ideal stem cell, quantity of stem cells, and delivery method. Research has shown increased uptake and function of stem cells when used with TMR.

The future is very bright for the treatment of CAD. The exciting leap forward of CABG combined with TMR and stem cells will lead to not only complete revascularization but neorevascularization as well. This can reduce repeat procedures, re-hospitalizations, and further coronary events. This can also lead to improvements in ejection fraction, exercise tolerance, and quality of life. Going forward the treatment of mild to moderate CAD will be PCI and moderate to severe CAD will be CABG/ TMR/stem cells.

Copyright: (C2015 Pirris J. This is an open-access article distributed under the terms of the Creative Commons Attribution License, which permits unrestricted use, distribution, and reproduction in any medium, provided the original author and source are credited. 